\title{
Anatomic single-bundle anterior cruciate ligament reconstruction using a calcium phosphate-hybridized tendon graft: a randomized controlled trial with 2 years of follow-up
}

Hirotaka Mutsuzaki ${ }^{*}$, Tomonori Kinugasa ${ }^{2}$, Kotaro Ikeda ${ }^{2}$ and Masataka Sakane ${ }^{3}$

\begin{abstract}
Background: To improve tendon-to-bone healing in anterior cruciate ligament (ACL) reconstruction, a novel technique via calcium phosphate (CaP)-hybridized tendon graft using an alternate soaking process was developed. The purpose of this study was to evaluate the clinical results of anatomic single-bundle ACL reconstruction using the $\mathrm{CaP}$-hybridized tendon graft with up to 2 years follow-up, and compare the outcome with conventional $\mathrm{ACL}$ reconstruction and preoperative data.
\end{abstract}

Methods: Ninety patients who required anatomic single-bundle ACL reconstruction were randomized to undergo either the CaP-hybridized tendon graft method (CaP group, $n=45$ ) or the conventional method (conventional group, $n=45$ ). At 1 and 2 years postoperatively, all patients were evaluated using KT-1000 arthrometry, pivot-shift test, International Knee Documentation Committee (IKDC) grade, Lysholm scale, and Tegner scale; at the same timepoints, bone tunnel enlargement was evaluated using computed tomography, and the tendon graft intensity was evaluated on magnetic resonance imaging. Tendon graft appearance was evaluated arthroscopically once after a period of up to 2 years postoperatively. Cases of re-rupture and adverse events were recorded in both groups.

Results: In both groups, the KT-1000 arthrometry, pivot-shift test, IKDC grade, and Lysholm scale results at 1 and 2 years postoperatively were superior to preoperative data; these results did not significantly differ between groups at either timepoint. The rate of increase of the cross-sectional area of the femoral bone tunnel in the CaP group was smaller than that in the conventional group at 1 year postoperatively. The other results did not significantly differ between the two groups at any timepoint. There were two cases of re-rupture in the CaP group, and six cases of re-rupture in the conventional group. There were no adverse events during follow-up in either group.

Conclusions: Anatomic single-bundle ACL reconstruction using a CaP-hybridized tendon graft was safe, and resulted in improved clinical outcomes at 2 years postoperatively compared with preoperative data; the outcomes were comparable with the conventional method. A longer follow-up is needed to clarify the clinical effects of the CaP-hybridized tendon graft in anatomic single-bundle ACL reconstruction.

Trial registration: UMIN, UMIN000019788 Registered 14 November 2015—Retrospectively registered.

Keywords: Anatomic single-bundle anterior cruciate ligament reconstruction, Calcium phosphate hybridization, Clinical result, Bone tunnel enlargement, A randomized controlled trial

\footnotetext{
* Correspondence: mutsuzaki@ipu.ac.jp

'Department of Orthopaedic Surgery, Ibaraki Prefectural University of Health

Sciences, 4669-2 Ami Ami-machi, Inashiki-gun, Ibaraki 300-0394, Japan

Full list of author information is available at the end of the article
}

(c) The Author(s). 2018 Open Access This article is distributed under the terms of the Creative Commons Attribution 4.0 International License (http://creativecommons.org/licenses/by/4.0/), which permits unrestricted use, distribution, and reproduction in any medium, provided you give appropriate credit to the original author(s) and the source, provide a link to the Creative Commons license, and indicate if changes were made. The Creative Commons Public Domain Dedication waiver (http://creativecommons.org/publicdomain/zero/1.0/) applies to the data made available in this article, unless otherwise stated. 


\section{Background}

After anterior cruciate ligament (ACL) reconstruction using a soft tissue graft, only fibrous tissue that was mechanically inferior was noted between the grafted tendon-bone interface [1-3]. To improve tendon-to-bone healing, a novel technique via calcium phosphate $(\mathrm{CaP})$-hybridized tendon graft using an alternate soaking process was developed [4]. We consider that firm tendon-to-bone healing in ACL reconstruction can improve clinical results and prevent bone tunnel enlargement. Using the CaP-hybridized tendon graft, direct bonding between the grafted tendon and the newly formed bone without scar tissue formation at 2 to 3 weeks after ACL reconstruction in rabbits was observed [5, 6]. In the $\mathrm{CaP}$ group, better anterior knee stability and greater in situ forces in the graft under applied anterior tibial loads were found at 1 year after non-anatomic ACL reconstruction in goats compared with an untreated tendon graft [3]. In a clinical trial, the CaP-hybridized tendon graft in non-anatomic single-bundle ACL reconstruction improved anterior knee stability at 1 and 2 years postoperatively, and reduced the bone tunnel enlargement in both tunnels at 1 year postoperatively compared with the conventional method [7].

In anatomic single-bundle ACL reconstruction using the CaP-hybridized tendon graft, greater in-situ forces in the graft under applied anterior tibial loads were found at 6 months postoperatively in goats compared with an untreated tendon graft [8]. A clinical trial showed that using a CaP-hybridized tendon graft reduced bone tunnel enlargement on the femoral side at 1 year after anatomic single-bundle ACL reconstruction compared with an untreated tendon graft, and the clinical data were equal in both groups [9]. However, the clinical trial included bilateral ACL reconstruction cases, and the follow-up period was only 1 year. Therefore, it was not possible to accurately compare the clinical results.

Therefore, the purpose of the present study was to prospectively evaluate the clinical results of unilateral anatomic single-bundle ACL reconstruction using a CaP-hybridized tendon graft during 2 years of follow-up in comparison with the conventional method and with preoperative data. We hypothesized that anatomic single-bundle ACL reconstruction using the CaP-hybridized tendon graft would result in equivalent postoperative clinical results to the conventional method, and would reduce femoral bone tunnel enlargement compared with the conventional method.

\section{Methods}

Between July 2011 and December 2015, a total of 90 patients who were scheduled to undergo arthroscopically assisted unilateral anatomic single-bundle ACL reconstruction using a hamstring tendon graft were enrolled in the present study. Patients were randomized to undergo either the CaP-hybridized tendon graft method
(CaP group, $n=45$ ) or the conventional method (conventional group, $n=45)$. The randomization was done according to the days of the week when the patients first visited the outpatient. All patients were followed up for a minimum of 2 years. The ethics committee of Ichihara Hospital reviewed and approved the study (approval number: 1101). Informed consent was obtained from the enrolled patients. The study protocol was registered with the University hospital Medical Information Network Clinical Trials Registry (UMIN000019788). We excluded revision cases, multi-ligamentous surgery cases, and bilateral ACL reconstruction cases. All ACL reconstructions were performed by two experienced surgeons (T.K. and H.M.). The patient characteristics did not differ between the two groups regarding age, sex, height, weight, operative findings of meniscal injury, and duration from injury to operation (Table 1). Meniscal pathology was confirmed by arthroscopic examination. In the $\mathrm{CaP}$ group, 4 medial and 6 lateral menisci were repaired, and 11 medial and 13 lateral menisci underwent partial resection; in the conventional group, 6 medial and 6 lateral menisci were repaired, and 1 medial and 12 lateral menisci underwent partial resection.

\section{Surgical procedure}

The surgical procedure was similar to that used in our previous study [9]. Briefly, after arthroscopic evaluation and treatment of associated lesions, the semitendinous tendon alone, or both the semitendinous and gracilis tendons, were harvested and used as multi-stranded grafts. The tendon graft was hooked to the TightRope $\mathrm{RT}^{\circledast}$ (Arthrex, Naples, FL, USA) on the femoral side. The free ends were whipstitched with FiberWire ${ }^{\bullet}$ 2 (Arthrex) on the tibial side. The length and diameter of the tendon grafts were $50-70 \mathrm{~mm}$ and $6.0-10.0 \mathrm{~mm}$, respectively. Both the femoral and tibial bone tunnels were anatomically created at the tibial and femoral

Table 1 Patient characteristics

\begin{tabular}{llll}
\hline & $\begin{array}{l}\text { CaP group } \\
(n=45)\end{array}$ & $\begin{array}{l}\text { Conventional } \\
\text { group }(n=45)\end{array}$ & $P$ value \\
\hline Age (years) & $27.1 \pm 11.1$ & $22.9 \pm 10.6$ & 0.072 \\
Sex (males/females) & $21 / 24$ & $26 / 19$ & 0.294 \\
Height (cm) & $166.2 \pm 8.3$ & $167.3 \pm 7.9$ & 0.517 \\
$\begin{array}{l}\text { Weight (kg) } \\
\begin{array}{l}\text { Operative findings of } \\
\text { meniscal injury (MM/LM) }\end{array}\end{array}$ & $15 / 19$ & $7 / 18$ & 0.261 \\
$\begin{array}{l}\text { Duration from injury to } \\
\text { operation (months) }\end{array}$ & $7.9 \pm 20.0$ & $2.1 \pm 2.2$ & 0.062 \\
\hline
\end{tabular}

The CaP group underwent single-bundle anterior cruciate ligament reconstruction with a calcium phosphate-hybridized graft, while the conventional group underwent reconstruction with a conventional tendon graft

Results are given as the mean $\pm \mathrm{SD}$

$M M$ medial meniscus, $L M$ lateral meniscus 
insertions of the ACL using the outside-in tunnel technique. A 5- to 15-mm-long femoral socket was created according to the length of the grafted tendon. The graft was fixed on the lateral femoral cortex, and then fixed to a screw and washer on the tibial side with an initial tension of $10 \mathrm{~N}$ using a tension meter at $20^{\circ}$ of knee flexion.

\section{Intraoperative calcium phosphate hybridization method}

The intraoperative CaP hybridization method was similar to that used in our previous study [7, 9]. After graft preparation, the intraarticular portion of the tendon graft was covered with the sleeve of a rubber glove tied on each side with nonabsorbable sutures to prevent $\mathrm{CaP}$ hybridization of the intraarticular portion $[7,9]$. Then, the tendon graft was soaked in a calcium solution $(100 \mathrm{mM} \mathrm{CaCl} 2+30 \mathrm{mM}$ L-histidine, $\mathrm{pH}$ 7.4, $280 \mathrm{mOsm} / \mathrm{l}, 20^{\circ} \mathrm{C}$ ) for $30 \mathrm{~s}$. The grafts were subsequently soaked in a $\mathrm{NaHPO}_{4}$ solution $(116.4 \mathrm{mM}$ $\mathrm{NaH}_{2} \mathrm{PO}_{4}: 128.7 \mathrm{mM} \mathrm{Na} \mathrm{HPO}_{4}: 12 \mathrm{H}_{2} \mathrm{O}=15 \%: 85 \%$, pH 7.4, $280 \mathrm{mOsm} / \mathrm{l}, 20^{\circ} \mathrm{C}$ ) for $30 \mathrm{~s}$. Before each soaking, the grafts were washed in a saline solution. This cycle was repeated ten times $[7,9]$.

\section{Postoperative rehabilitation}

Postoperative rehabilitation was similar to that performed in our previous study [9]. After surgery, the knee was immobilized at $20^{\circ}$ flexion with a removable postoperative brace for 1 week. Range of motion exercise and partial weightbearing was started at 1 week postoperatively, and full weightbearing walking was allowed at 3 weeks postoperatively. Running was allowed at 3 months postoperatively, and return to sports was allowed after 6-12 months. The postoperative rehabilitation was the same for all patients, even those who received partial meniscectomy or meniscal repair.

\section{Clinical evaluations}

The patients were assessed preoperatively, and at 1 and 2 years postoperatively. Anterior knee laxity was assessed using the KT-1000 arthrometer (MEDmetric, San Diego, CA, USA) at manual maximum anterior tibial load. The pivot-shift test, the Tegner scale [10], the International Knee Documentation Committee (IKDC) grade [11], and the Lysholm scale [10] were also assessed. Preoperative Tegner scale data were assessed using data obtained before ACL injury. Cases of re-rupture and adverse events (including infection, fracture, cancer, severe pain, contracture, etc.) were recorded in both groups.

\section{Computed tomography analysis}

All patients underwent computed tomography (CT) evaluation at postoperative 1 week, 1 year, and 2 years. The full evaluation was performed independently by a radiologist who was blinded to the study grouping. CT scans (voltage $80 \mathrm{kV}$; Activion 16; Toshiba Medical Systems, Otawara, Japan) were performed with the knee in full extension to assess the femoral and tibial bone tunnels. Initial volume acquisition was made with $2-\mathrm{mm}$ cuts from $10 \mathrm{~cm}$ above the femoral tunnel to $10 \mathrm{~cm}$ below the tibial tunnel. Three-dimensional images were reconstructed using a Virtual Place Lexus workstation (AZE, Tokyo, Japan) [7, 12, 13]. As the joint aperture site of the bone tunnels is reportedly enlarged after ACL reconstruction $[12,13]$, we evaluated both the femoral and the tibial tunnels at the aperture. The two groups were compared regarding the tunnel enlargement rates of the cross-sectional areas (CSAs) at the apertures of both the femoral and tibial tunnels from 1 week to 1 year postoperatively, and from 1 week to 2 years postoperatively.

The bone tunnel enlargement evaluation was performed using a similar method to that used in our previous study [9]. The tunnels were cut along planes perpendicular to the long axes. We measured the tunnel CSAs of the femur and tibia at the sites closest to the joint aperture. The increase in tunnel CSA was calculated as follows: CSA increase rate $(\%)=($ CSA at 1 year or 2 year - CSA at 1 week) $\times 100 /$ CSA at 1 week.

\section{Magnetic resonance imaging evaluation}

The magnetic resonance imaging (MRI) evaluation was similar to that described previously [7]. MRI evaluation was performed to analyze the intensity of the tendon graft, which is a measure of graft remodeling, at 1 and 2 years postoperatively. The tendon grafts of the mid-substance area were characterized as low intensity, isointensity, or high intensity by proton density imaging in the sagittal, coronal, and axial planes with 3.0-Tesla MRI (MAGNETOM Verio Dot; Siemens Healthineers, Earlangen, Germany) [7, 14]. The numbers belonging to each characterization were investigated and compared between both groups.

\section{Arthroscopic evaluation}

The arthroscopic evaluation method was similar to that used in our previous report [7]. Second-look arthroscopic examination was performed once at 1 to 2 years postoperatively to analyze the synovium coverage of the tendon graft, which provides an indication of revascularization. The synovium coverage of each reconstructed graft was graded as A (completely covered), B (partially covered), or $C$ (almost uncovered) [7, 15]. The numbers belonging to each grade were investigated and compared between both groups.

\section{Statistical analyses}

The Student's $t$ test was used to analyze the results of KT-1000 arthrometry, Lysholm scores, and CT analyses 
between each group at the same timepoint. The chi-square for independence test was used to analyze the results of the pivot-shift test, Tegner scores, IKDC results, MRI evaluation, and arthroscopic appearance between groups at the same timepoint. The paired $t$ test was used to analyze the KT-1000 arthrometry and Lysholm scores between preoperatively and 1 year postoperatively, and between preoperatively and 2 years postoperatively within the same group. The Wilcoxon signed-ranks test was used to analyze the pivot-shift test results, Tegner scores, and IKDC results between preoperatively and 1 year postoperatively, and between preoperatively and 2 years postoperatively within the same group. Differences were considered significant at $P<0.05$.

\section{Results}

A flow-chart of the present study is shown in Fig. 1.

Clinical results are summarized in Table 2. There were two cases of re-rupture in the CaP group (one case occurred within 1 year, and one case occurred more than 1 year postoperatively), and six cases of re-rupture in the conventional group (four cases within 1 year, and two cases after more than 1 year) in 2 years of follow-up. In

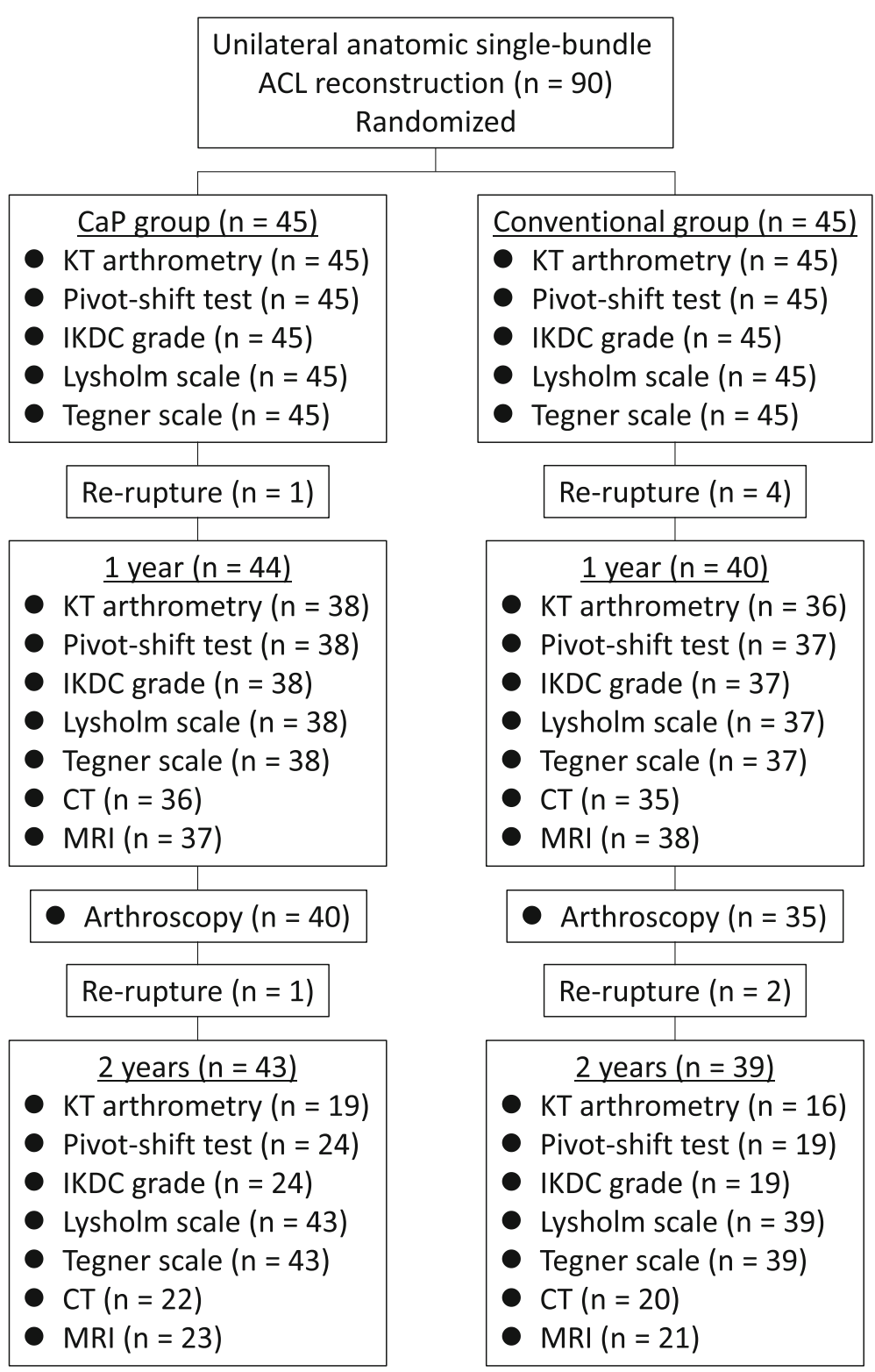

Fig. 1 Flowchart of the study. The CaP group underwent single-bundle anterior cruciate ligament reconstruction with a calcium phosphatehybridized graft, while the conventional group underwent reconstruction with a conventional tendon graft. ACL anterior cruciate ligament, IKDC International Knee Documentation Committee 
Table 2 Clinical results

\begin{tabular}{|c|c|c|c|}
\hline & CaP group & Conventional group & $P$ value \\
\hline \multicolumn{4}{|l|}{ Re-ruptured cases ( $n(\%))$} \\
\hline 1 year postoperatively & $1(2.2 \%)$ & $4(8.9 \%)$ & 0.167 \\
\hline 2 years postoperatively & $1(2.2 \%)$ & $2(4.4 \%)$ & 0.557 \\
\hline Total & $2(4.4 \%)$ & $6(13.3 \%)$ & 0.138 \\
\hline \multicolumn{4}{|c|}{ Anterior knee laxity measured by KT-1000 arthrometry (mm) } \\
\hline Preoperative & $9.2 \pm 3.2(n=45)$ & $10.1 \pm 2.5(n=45)$ & 0.139 \\
\hline 1 year postoperatively & $0.7 \pm 1.1(n=38)+$ & $0.4 \pm 1.0(n=36) \dagger$ & 0.323 \\
\hline 2 years postoperatively & $0.9 \pm 1.5(n=19) \dagger$ & $0.1 \pm 0.8(n=16) \dagger$ & 0.094 \\
\hline \multicolumn{4}{|c|}{ Pivot-shift test: $0,1+, 2+, 3+(n)$} \\
\hline Preoperative & $0,8,37,0(n=45)$ & $0,6,39,0(n=45)$ & 0.561 \\
\hline 1 year postoperatively & $36,1,1,0(n=38)+$ & $35,2,0,0(n=37)+$ & 0.513 \\
\hline 2 years postoperatively & $22,2,0,0(n=24)+$ & $19,0,0,0(n=19)+$ & 0.198 \\
\hline \multicolumn{4}{|l|}{ IKDC grade: A, B, C, D (n) } \\
\hline Preoperative & $0,2,27,16(n=45)$ & $0,0,28,17(n=45)$ & 0.359 \\
\hline 1 year postoperatively & $35,2,1,0(n=38)+$ & $34,3,0,0(n=37)+$ & 0.548 \\
\hline 2 years postoperatively & $20,4,0,0(n=24) \dagger$ & $19,0,0,0(n=19)+$ & 0.062 \\
\hline \multicolumn{4}{|l|}{ Lysholm scale (points) } \\
\hline Preoperative & $54.6 \pm 9.6(n=45)$ & $51.7 \pm 7.4(n=45)$ & 0.117 \\
\hline 1 year postoperatively & $98.2 \pm 2.9(n=38)+$ & $98.6 \pm 2.2(n=37)+$ & 0.464 \\
\hline 2 years postoperatively & $98.2 \pm 4.5(n=43) \dagger$ & $99.5 \pm 1.8(n=39) \dagger$ & 0.110 \\
\hline \multicolumn{4}{|c|}{ Tegner scale: $0,1,2,3,4,5,6,7,8,9,10(n)$} \\
\hline Preoperative (preinjury) & $0,0,0,2,3,3,18,10,1,8,0(n=45)$ & $0,0,0,0,2,0,14,12,1,16,0(n=45)$ & 0.201 \\
\hline 1 year postoperatively & $0,0,0,3,7,9,10,6,0,3,0(n=38) \dagger$ & $0,0,0,0,4,9,14,4,0,6,0(n=37) \dagger$ & 0.319 \\
\hline 2 years postoperatively & $0,0,0,3,2,6,14,10,1,7,0(n=43)$ & $0,0,0,0,2,2,13,9,0,13,0(n=39)$ & 0.260 \\
\hline
\end{tabular}

Results are given as the mean \pm SD

Significant difference between the two groups $(P<0.05)$; $†$ significant difference compared with preoperative value $(P<0.05)$

IKDC International Knee Documentation Committee

The CaP group underwent single-bundle anterior cruciate ligament reconstruction with a calcium phosphate-hybridized graft, while the conventional group underwent reconstruction with a conventional tendon graft

the two cases of re-rupture in the CaP group, the femoral socket depths were $10 \mathrm{~mm}$ and $12 \mathrm{~mm}$, the harvested tendons were semitendinous tendons alone, and the graft diameters were $8 \mathrm{~mm}$ and $8.5 \mathrm{~mm}$ in femoral side and both $9 \mathrm{~mm}$ in tibial side, respectively. The clinical results were evaluated after excluding the re-ruptured cases. Within each group, the anterior knee laxity at 1 and 2 years postoperatively was significantly lesser than preoperatively; there was also a significant difference in pivot-shift test and IKDC results at 1 and 2 years postoperatively compared with preoperatively, and the Lysholm scale at 1 and 2 years postoperatively was significantly larger than preoperatively. There was no significant difference between the $\mathrm{CaP}$ and conventional groups regarding anterior knee laxity, pivot-shift test, Tegner scale, IKDC grade, Lysholm scale, and re-rupture rate at the same timepoint. There were no adverse events at both follow-up timepoints in either group.
Bone tunnel enlargement results are summarized in Table 3. The CSA increase rate in the femoral bone tunnel of the CaP group was significantly smaller than that of the conventional group at 1 year postoperatively. The other results did not significantly differ between groups at either follow-up timepoint.

The results regarding the intensity of the tendon graft and the arthroscopic appearance are summarized in Table 4. There was no significant difference between groups in the intensity of the tendon graft or the arthroscopic appearance at both follow-up timepoints.

\section{Discussion}

The main findings of the present study were that anatomic single-bundle ACL reconstruction using the CaP-hybridized tendon graft was safe, resulted in improved clinical results at 1 and 2 years postoperatively compared with preoperatively, and was as stable as the conventional method. The femoral bone tunnel increase 
Table 3 Postoperative bone tunnel enlargement

\begin{tabular}{llll}
\hline & CaP group & Conventional group & $P$ value \\
\hline Femur & & & \\
1 year (\%) & $24.1 \pm 49.8(n=36)$ & $63.8 \pm 66.1(n=35)$ & $0.006^{*}$ \\
2 years (\%) & $13.4 \pm 58.1(n=22)$ & $41.8 \pm 66.7(n=20)$ & 0.148 \\
Tibia & & & \\
1 year (\%) & $17.3 \pm 24.5(n=36)$ & $18.9 \pm 25.5(n=35)$ & 0.788 \\
2 years (\%) & $6.4 \pm 31.7(n=22)$ & $1.5 \pm 22.5(n=20)$ & 0.573 \\
\hline
\end{tabular}

Results are given as the mean $\pm S D$

*Significant difference between the two groups $(P<0.05)$

The CaP group underwent single-bundle anterior cruciate ligament reconstruction with a calcium phosphate-hybridized graft, while the conventional group underwent reconstruction with a conventional tendon graft

rate at 1 year postoperatively in the $\mathrm{CaP}$ group was smaller than that in the conventional group. The postoperative intensity of the tendon graft and arthroscopic appearance in the $\mathrm{CaP}$ group were equivalent to those in the conventional group. The $\mathrm{CaP}$ hybridization technique is simple, and can be applied any patients ACL reconstruction is required. Moreover, the cost is inexpensive because it only involves the solutions preparation.

The postoperative clinical results in the $\mathrm{CaP}$ group were improved compared with preoperatively, and were similar to the results after conventional anatomic single-bundle ACL reconstruction. Moreover, no adverse events occurred in the follow-up period. Therefore, the CaP-hybridized tendon graft safely and effectively improved the postoperative clinical results in anatomic single-bundle ACL reconstruction. In non-anatomic single-bundle ACL reconstruction, the clinical results at 1 and 2 years postoperatively in the CaP group were reportedly superior to the results after reconstruction via the conventional method [7]. However, in the present study, the clinical results at 1 and 2 years postoperatively were equal in both groups. This may be because anatomic ACL reconstruction results in superior stability

Table 4 Postoperative MRI findings and arthroscopic appearance

\begin{tabular}{llll}
\hline & CaP group & Conventional group & $P$ value \\
\hline Graft intensity on MRI: low, iso, high & & \\
1 year & $20,17,0(n=37)$ & $16,21,1(n=38)$ & 0.396 \\
2 years & $16,6,1(n=23)$ & $19,2,0(n=21)$ & 0.205 \\
$\begin{array}{l}\text { Arthroscopic } \\
\text { appearance: A, B, C }\end{array}$ & $25,12,3(n=40)$ & $22,9,4(n=35)$ & 0.806 \\
\hline
\end{tabular}

Significant difference between the two groups $(P<0.05)$ The CaP group underwent single-bundle anterior cruciate ligament reconstruction with a calcium phosphate-hybridized graft, while the conventional group underwent reconstruction with a conventional tendon graft

The arthroscopic appearance of the synovium coverage of each graft was graded as A (completely covered), B (partially covered), or C (almost uncovered) compared with non-anatomic ACL reconstruction [16, 17], and the follow-up period was short in the present study. Therefore, a longer follow-up is needed to clarify the clinical effects of the CaP-hybridized tendon graft in anatomic single-bundle ACL reconstruction.

In the present study, the CaP-hybridized tendon graft resulted in a reduced femoral bone tunnel enlargement compared with the conventional method at 1 year postoperatively; the lack of data at 2 years postoperatively in both groups may be the reason that no significant difference was detected between groups at 2 years. The articular ends of the bone tunnels are enlarged by bone resorption, which is associated with graft-tunnel motion after ACL reconstruction with suspensory fixation [18, 19], and synovial fluid cytokines cause delayed tendon-to-bone healing at the joint aperture site of the bone tunnel [20]. The CaP-hybridized tendon graft enhances bone formation on the surface of the tendon graft in the bone tunnel compared with the untreated tendon graft $[5,6]$. Therefore, more new bone can form in the femoral tunnels in the $\mathrm{CaP}$ method than the conventional method because of its osteogenic effect. Previous studies in the goat revealed that the CaP-hybridized tendon graft enhanced tendon-to-bone healing in the cartilage layer, and enhanced the new formation of bone near the joint aperture of the femoral and tibial bone tunnels $[3,8]$. The cartilage layer can act as a shock absorber against graft-tunnel motion, and as a protective wall against synovial fluid when it is anchored near the joint aperture. The anchoring provided by new bone formation and the cartilage layer in the $\mathrm{CaP}$ group may effectively prevent the progression of bone tunnel enlargement. Longer follow-up is needed to investigate the bone tunnel enlargement, and the association between bone tunnel enlargement and knee stability. In the present study, the $\mathrm{CaP}$ method prevented bone tunnel enlargement, especially on the femoral side. Bone tunnel enlargement was particularly evident on the femoral side in conventional ACL reconstruction in a previous study [21]. Greater shear stress can occur at the interface in the femoral bone tunnel than on the tibial side, comprising both longitudinal micromotion (bungee effect) and transverse micromotion (windshield wiper effect) [22].

The tendon grafts in the two groups in the present study had a similar intensity on MRI and a similar arthroscopic appearance. The CaP hybridization method did not cause complications in the grafted tendons on MRI and arthroscopic analysis. This indicates that the CaP-hybridized tendon graft is safe for use in clinical practice. The intensity of tendon grafts on MRI and the arthroscopic appearance of the tendon grafts indicate the graft maturation process (ligamentization and revascularization) after ACL reconstruction [23, 24]. We 
believe that firm anchoring is important for maturation of the tendon graft, as firm anchoring can lead to the application of appropriate mechanical stress on the tendon graft. MRI analysis at more than 2 years after ACL reconstruction may be required to more accurately evaluate the ligamentization and revascularization of the tendon grafts in the two groups.

The present study had some limitations. The evaluations were only performed at three timepoints, and the follow-up period was short. Furthermore, there were missing data. Longer follow-up with a complete dataset is needed to investigate any increase in instability, bone tunnel enlargement, and graft maturation. Moreover, it is necessary to evaluate the relationship between bone tunnel enlargement, graft maturation, and knee instability.

\section{Conclusions}

Anatomic single-bundle ACL reconstruction using the CaP-hybridized tendon graft is safe, and results in improved postoperative clinical results compared with preoperative data during 2 years of follow-up. The postoperative clinical results in the $\mathrm{CaP}$ group were as stable as those in the conventional group. The CaP-hybridized tendon graft can reduce postoperative femoral bone tunnel enlargement compared with the untreated tendon graft. A longer follow-up is needed to clarify the clinical effects of the CaP-hybridized tendon graft in anatomic single-bundle ACL reconstruction.

\section{Abbreviations}

ACL: Anterior cruciate ligament; CaP: Calcium phosphate; CSA: Crosssectional area; CT: Computed tomography; IKDC: International Knee Documentation Committee; MRI: Magnetic resonance imaging

\section{Acknowledgments}

We thank Kelly Zammit, BVSc, from Edanz Group (www.edanzediting.com/ac), for editing a draft of this manuscript.

\section{Funding}

This work was supported by the Japan Sports Medicine Foundation, 2015.

\section{Availability of data and materials}

The datasets generated and/or analyzed during the current study are available from the corresponding author by reasonable request.

\section{Authors' contributions}

HM and TK conceived the study, and participated in its design and coordination. HM and TK performed the operations. HM analyzed the data and drafted the manuscript. All authors interpreted the data and participated in drafting the text and tables. All authors read and approved the final manuscript.

\section{Ethics approval and consent to participate}

The ethics committee of Ichihara Hospital reviewed and approved this retrospective study (approval number 1101).

\section{Consent for publication}

Not applicable.

\section{Competing interests}

The authors declare that they have no competing interests.

\section{Publisher's Note}

Springer Nature remains neutral with regard to jurisdictional claims in published maps and institutional affiliations.

\section{Author details}

${ }^{1}$ Department of Orthopaedic Surgery, Ibaraki Prefectural University of Health Sciences, 4669-2 Ami Ami-machi, Inashiki-gun, Ibaraki 300-0394, Japan. ${ }^{2}$ Department of Orthopaedic Surgery, Ichihara Hospital, 3681 Oozone, Tsukuba, Ibaraki 300-3295, Japan. ${ }^{3}$ Department of Orthopaedic Surgery, Tsukuba Gakuen Hospital, 2573-1 Kamiyokoba, Tsukuba, Ibaraki 305-0854, Japan.

Received: 20 April 2018 Accepted: 18 December 2018 Published online: 29 December 2018

\section{References}

1. Grana WA, Egle DM, Mahnken R, Goodhart CW. An analysis of autograft fixation after anterior cruciate ligament reconstruction in a rabbit model. Am J Sports Med. 1994;22:344-51.

2. Nebelung W, Becker R, Urbach D, Röpke M, Roessner A. Histological findings of tendon-bone healing following anterior cruciate ligament reconstruction with hamstring grafts. Arch Orthop Trauma Surg. 2003;123:158-63.

3. Mutsuzaki H, Sakane M, Fujie H, Hattori S, Kobayashi H, Ochiai N. Effect of calcium phosphate-hybridized tendon graft on biomechanical behavior in anterior cruciate ligament reconstruction in a goat model: novel technique for improving tendon-bone healing. Am J Sports Med. 2011;39:1059-66.

4. Taguchi T, Kishida A, Akashi M. Hydroxyapatite formation on/in hydrogels using a novel alternate soaking process. Chem Lett. 1998;8:711-2.

5. Mutsuzaki H, Sakane M, Nakajima H, Ito A, Hattori S, Miyanaga Y, Ochiai N, Tanaka J. Calcium-phosphate-hybridized tendon directly promotes regeneration of tendon-bone insertion. J Biomed Mater Res A. 2004;70A: 319-27.

6. Mutsuzaki H, Sakane M, Ito A, Nakajima H, Hattori S, Miyanaga Y, Tanaka J, Ochiai N. The interaction between osteoclast-like cells and osteoblasts mediated by nanophase calcium phosphate-hybridized tendons. Biomaterials. 2005;26:1027-34.

7. Mutsuzaki H, Kanamori A, Ikeda K, Hioki S, Kinugasa T, Sakane M. Effect of calcium phosphate-hybridized tendon graft in anterior cruciate ligament reconstruction: a randomized controlled trial. Am J Sports Med. 2012;40:1772-80.

8. Mutsuzaki H, Fujie H, Nakajima H, Fukagawa M, Nomura S, Sakane M. Effect of calcium phosphate hybridized tendon graft in anatomical single-bundle ACL reconstruction in goats. Orthop J Sports Med. 2016;4: 2325967116662653.

9. Mutsuzaki H, Kinugasa T, Ikeda K, Sakane M. Calcium phosphate-hybridized tendon grafts reduce femoral bone tunnel enlargement in anatomic singlebundle ACL reconstruction. Knee Surg Sports Traumatol Arthrosc. 2018;26:500-7.

10. Tegner $Y$, Lysholm J. Rating systems in the evaluation of knee ligament injuries. Clin Orthop Relat Res. 1985;198:43-9.

11. Hefti F, Muller W, Jakob RP, Staubli HU. Evaluation of knee ligament injuries with the IKDC form. Knee Surg Sports Traumatol Arthrosc. 1993;1:226-34.

12. Taketomi S, Inui H, Sanada T, Yamagami R, Tanaka S, Nakagawa T. Eccentric femoral tunnel widening in anatomic anterior cruciate ligament reconstruction. Arthroscopy. 2014:30:701-9.

13. Tachibana Y, Mae T, Shino K, Kanamoto T, Sugamoto K, Yoshikawa H, Nakata K. Morphological changes in femoral tunnels after anatomic anterior cruciate ligament reconstruction. Knee Surg Sports Traumatol Arthrosc. 2015:23:3591-600

14. Endele D, Jung C, Becker U, Bauer G, Mauch F. Anterior cruciate ligament reconstruction with and without computer navigation: a clinical and magnetic resonance imaging evaluation 2 years after surgery. Arthroscopy. 2009;25:1067-74.

15. Kondo E, Yasuda K. Second-look arthroscopic evaluations of anatomic double-bundle anterior cruciate ligament reconstruction: relation with postoperative knee stability. Arthroscopy. 2007;23:1198-209.

16. Lim HC, Yoon YC, Wang JH, Bae JH. Anatomical versus nonanatomical single bundle anterior cruciate ligament reconstruction: a cadaveric study of comparison of knee stability. Clin Orthop Surg. 2012;4:249-55.

17. Riboh JC, Hasselblad V, Godin JA, Mather RC 3rd. Transtibial versus independent drilling techniques for anterior cruciate ligament 
reconstruction: a systematic review, meta-analysis, and meta-regression. Am J Sports Med. 2013;41:2693-702.

18. Rodeo SA, Kawamura S, Kim HJ, Dynybil C, Ying L. Tendon healing in a bone tunnel differs at the tunnel entrance versus the tunnel exit: an effect of graft-tunnel motion? Am J Sports Med. 2006;34:1790-800.

19. Rodeo SA, Kawamura S, Ma CB, Deng XH, Sussman PS, Hays P, Ying L. The effect of osteoclast activity on tendon-to-bone healing: an experimental study in rabbits. J Bone Joint Surg Am. 2007;89A:2250-9.

20. Berg EE, Pollard ME, Kang Q. Interarticular bone tunnel healing. Arthroscopy. 2001;17:189-95.

21. Feller JA, Webster KE. A randomized comparison of patellar tendon and hamstring tendon anterior cruciate ligament reconstruction. Am J Sports Med. 2003;31:564-73.

22. Paessler HH, Mastrokalos DS. Anterior cruciate ligament reconstruction using semitendinosus and gracilis tendons, bone patellar tendon, or quadriceps tendon-graft with press-fit fixation without hardware. A new and innovative procedure. Orthop Clin North Am. 2003:34:49-64.

23. Howell SM, Berns GS, Farley TE. Unimpinged and impinged anterior cruciate ligament grafts: MR signal intensity measurements. Radiology. 1991;179:639-43.

24. Bellelli A, Adriani E, Margheritini F, Camillieri G, Della Rocca C, Mariani PP. Synovial healing in reconstructed cruciate ligaments: our personal experience compared in single interventions and combined reconstructions. Radiol Med. 1999:98:454-61.

Ready to submit your research? Choose BMC and benefit from:

- fast, convenient online submission

- thorough peer review by experienced researchers in your field

- rapid publication on acceptance

- support for research data, including large and complex data types

- gold Open Access which fosters wider collaboration and increased citations

- maximum visibility for your research: over $100 \mathrm{M}$ website views per year

At $\mathrm{BMC}$, research is always in progress.

Learn more biomedcentral.com/submissions 